\title{
Improved Generalized Force Model considering the Comfortable Driving Behavior
}

\author{
De-Jie Xu, Bao-Hua Mao, Shao-Kuan Chen, and Yun Bai \\ MOE Key Laboratory for Urban Transportation Complex Systems Theory and Technology, Beijing Jiaotong University, \\ Beijing 100044, China
}

Correspondence should be addressed to De-Jie Xu; xudejie126@126.com

Received 17 November 2014; Revised 12 March 2015; Accepted 15 March 2015

Academic Editor: Sebastian Anita

Copyright (C) 2015 De-Jie Xu et al. This is an open access article distributed under the Creative Commons Attribution License, which permits unrestricted use, distribution, and reproduction in any medium, provided the original work is properly cited.

This paper presents an improved generalized force model (IGFM) that considers the driver's comfortable driving behavior. Through theoretical analysis, we propose the calculation methods of comfortable driving distance and velocity. Then the stability condition of the model is obtained by the linear stability analysis. The problems of the unrealistic acceleration of the leading car existing in the previous models were solved. Furthermore, the simulation results show that IGFM can predict correct delay time of car motion and kinematic wave speed at jam density, and it can exactly describe the driver's behavior under an urgent case, where no collision occurs. The dynamic properties of IGFM also indicate that stability has improved compared to the generalized force model.

\section{Literature Review and Introduction}

In recent several decades, lots of traffic models have been proposed by scholars to describe the complicated traffic phenomena. In essence, traffic models can be divided into two categories: macroscopic and microscopic models [1]. The former was proposed on the basis of hydrodynamics regarding the traffic flow as a flow of continuous medium, and the latter may focus more on each individual car, which mainly includes cellular automaton models [2-4] and carfollowing models [5-11]. The original car-following model was proposed by Reuschel and Pipes [12,13], and its basic assumption is that the driver can adjust the vehicular speed according to the velocity difference of two neighboring cars. Because the velocity difference is just considered in it, the case that only one car exits in the system is neglected, which causes that the model cannot describe the situation correctly which just had one car; besides, when the successive two vehicles travel at the same speed, the headway distance may be very close to each other; this does not conform to the reality. In order to overcome these shortages, Newell [14] presented another model, whose idea is that the velocity depends on the headway distance. So the velocity is expressed as a function of the headway.
In 1995, Bando et al. [15] developed the optimal velocity model (OVM), which has similar idea to the Newell's model, but it improves the optimal function and can be interpreted simply compared with the previous models, so it attracts many researchers' interests. The dynamic equation of OVM is as follows:

$$
\frac{\mathrm{d} v_{n}}{\mathrm{~d} t}(t)=\kappa\left[V\left(s_{n}(t)\right)-v_{n}(t)\right],
$$

where $v_{n}(t)$ is the velocity of $n$th car at time $t, \kappa$ is the sensitivity coefficient, $s_{n}(t)$ indicates the space headway, and $V\left(s_{n}(t)\right)$ denotes the optimal velocity.

Helbing and Tilch [16] calibrated the parameters of OVM applied to the field data, and they found out that the value of acceleration is unrealistically high. Therefore, they introduced the velocity difference term into the OVM and developed the generalized force model (GFM):

$$
\frac{\mathrm{d} v_{n}}{\mathrm{~d} t}=\kappa\left[V\left(s_{n}, v_{n}\right)-v_{n}\right]+\lambda \Theta\left(-\Delta v_{n}\right) \Delta v_{n},
$$

where $\kappa=1 / \tau_{n}$ is the sensitivity coefficient, $\tau_{n}$ is the acceleration time, and $V\left(s_{n}, v_{n}\right)$ is the optimal velocity function, which reads

$$
V\left(s_{n}, v_{n}\right)=v_{m}\left\{1-e^{-\left[s_{n}-s\left(v_{n}\right)\right] / R_{n}}\right\},
$$


TABLE 1: The values of $\delta t$ and $C_{j}$ in different models.

\begin{tabular}{lcc}
\hline Model & $\delta t(\mathrm{~s})$ & $C_{j}(\mathrm{~km} / \mathrm{h})$ \\
\hline $\operatorname{OVM}\left(\kappa=0.85 \mathrm{~s}^{-1}\right)$ & 1.60 & 16.65 \\
$\operatorname{GFM}\left(\kappa=0.41 \mathrm{~s}^{-1}\right)$ & 1.76 & 12.96 \\
$\operatorname{IGFM}\left(\kappa=0.25 \mathrm{~s}^{-1}\right)$ & 1.22 & 18.84 \\
\hline
\end{tabular}

where $v_{m}$ is the maximal desired velocity, $R_{n}$ is the range of the acceleration interaction. Consider

$$
s\left(v_{n}\right)=d_{n}+T_{n} v_{n},
$$

where $d_{n}$ is the minimal headway distance and $T_{n}$ is the safe time headway.

In order to avoid the vehicle collision, the velocity difference term is added into the GFM and it is only effective when the velocity of the following vehicle is larger than that of the leading vehicle; that is, if $\Delta v_{n} \leq 0, \Theta\left(-\Delta v_{n}\right)=1$. The sensitivity coefficient of velocity difference term is $\lambda$ and we call it acceleration term coefficient, which is given by

$$
\lambda=\frac{1}{\tau_{n}^{\prime}} e^{-\left[s_{n}-s\left(v_{n}\right)\right] / R_{n}^{\prime}},
$$

where $\tau_{n}^{\prime}$ denotes the braking time and $R_{n}^{\prime}$ can be interpreted as range of the braking interaction.

However, Jiang et al. [17] pointed out that kinematic wave speed at jam density and delay time of car motion of GFM are poor because of ignoring the effects of positive velocity differences. So, through considering both positive and negative velocity differences, they proposed a full velocity difference model (FVDM) that takes the sensitivity coefficient $\lambda$ as a constant.

Then Gong et al. $[18,19]$ found that FVDM would lead to a problem that the following vehicle may not brake even if the distance to the leading vehicle is extremely close, and they proposed an asymmetric full velocity difference model (AFVDM) that takes the asymmetric characteristic into account. Yu et al. [20] proposed a full velocity difference and acceleration model (FVDAM) by synthetically taking into account headway, velocity difference, and acceleration of the leading car; the following car in FVDAM can accelerate more quickly than that in FVDM.

For the above mentioned models, they have made a great progress in car-following theory. However, they have a problem that the maximum acceleration of leader starting from a standing state is unrealistically high. Synthesizing all the above models, we consider the variation of acceleration under the case that the leading car is initially at rest and unobstructed; then it starts up. The velocity difference is null in all the above models because the space distance is greater than the range of interaction, so the acceleration of the leading car is

$$
\frac{\mathrm{d} v}{\mathrm{~d} t}=\kappa\left[v_{m}-v(t)\right]
$$

From (6), the maximal acceleration of the leading car is $\kappa v_{m}$ and it only related to the sensitivity coefficient $\kappa$ of

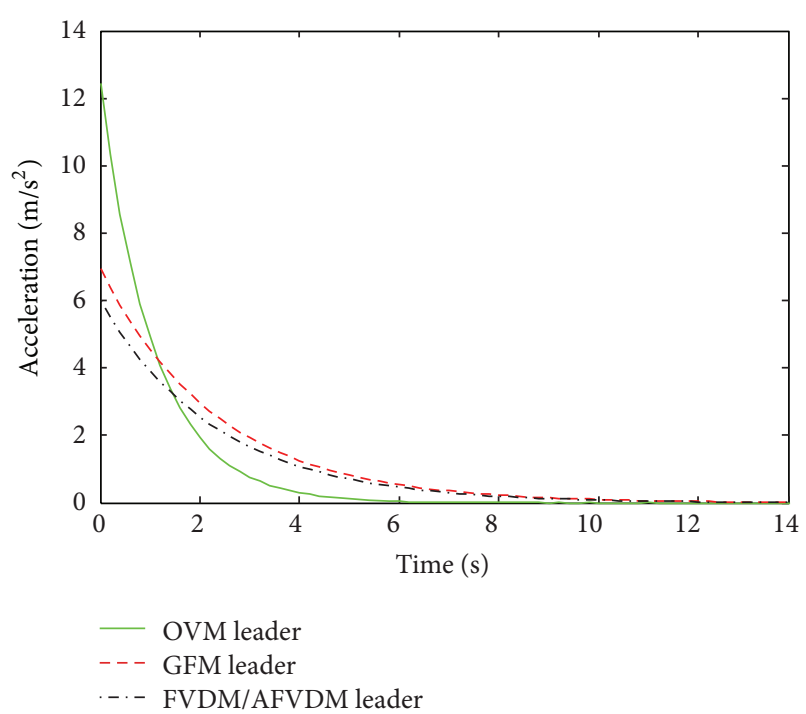

FIGURE 1: Acceleration of unobstructed leading car according to the different models.

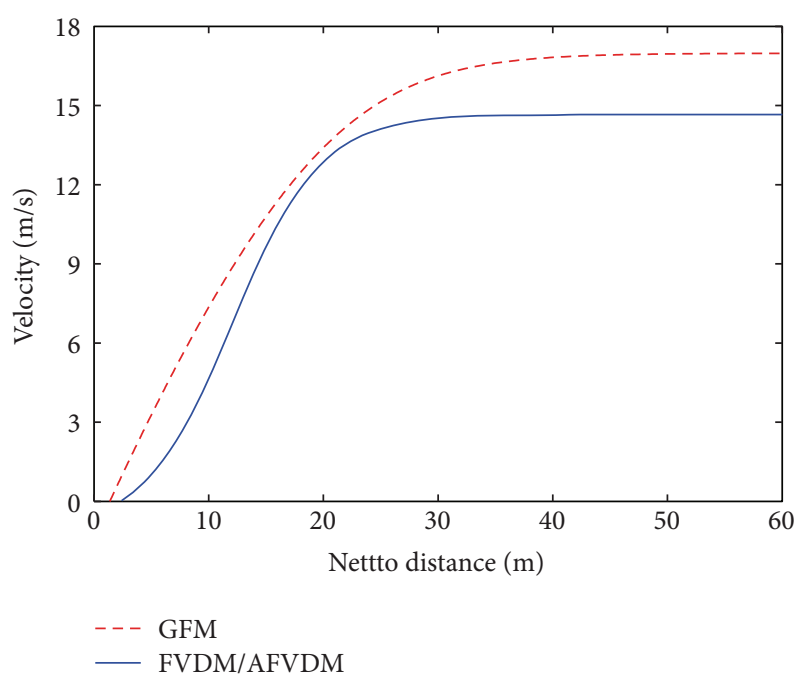

FIGURE 2: Velocity-distance relations of GFM, FVDM, and AFVDM in the stationary case.

different models (cf. Table 1 ) if $v_{m}$ is fixed. Figure 1 shows the acceleration variation of the leading car. We can see that the maximal accelerations are $12.46 \mathrm{~m} / \mathrm{s}^{2}$ in OVM and $6.96 \mathrm{~m} / \mathrm{s}^{2}$ in GFM. The optimal velocity functions in FVDM and AFVDM are different from that in GFM, as shown in Figure 2, so their maximal accelerations are $6.01 \mathrm{~m} / \mathrm{s}^{2}$. However, according to [16], the empirical acceleration is usually not greater than $4 \mathrm{~m} / \mathrm{s}^{2}$. Thus, the accelerations of OVM, GFM, FVDM, and AFVDM are unrealistically high.

In order to overcome the shortages of the previous models, we present an improved generalized model (IGFM) by introducing a velocity difference term based on the drivers' comfortable driving behavior. This contribution is organized as follows: the new model will be presented in 


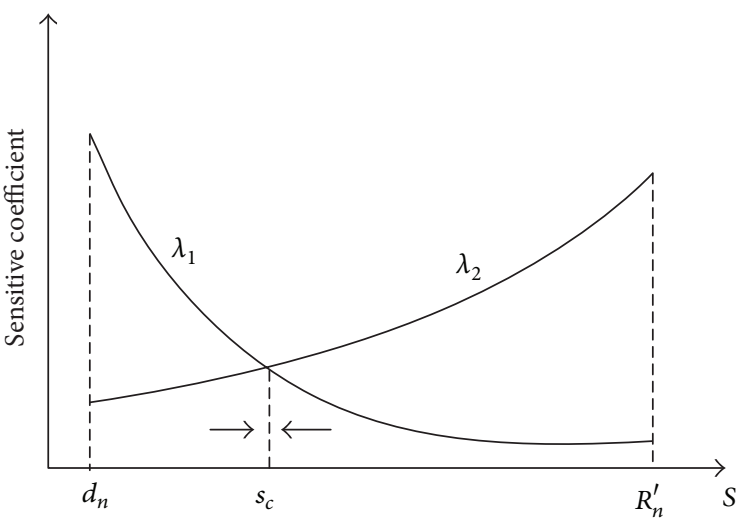

(a)

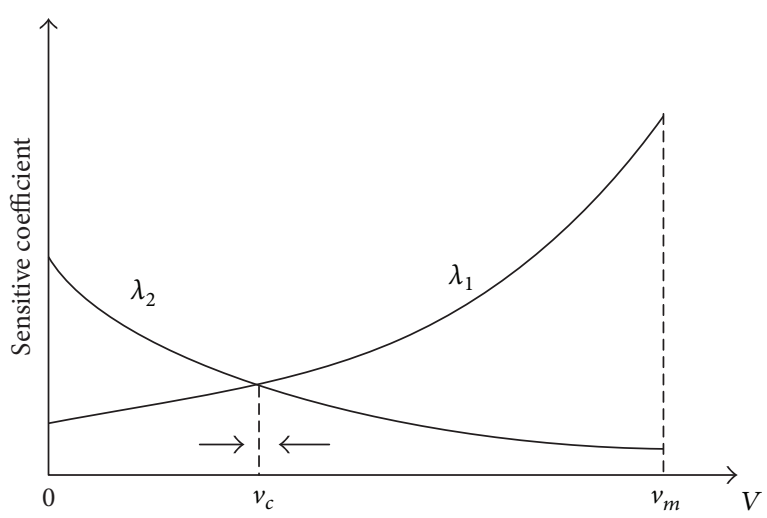

(b)

FIGURE 3: Relationship between the two sensitivity coefficients and (a) the space headway (b) and the velocity.

Section 2. Simulations and result discussions are carried out in Section 3. Finally, the conclusions are given in Section 4.

\section{The Improved Generalized Force Model}

2.1. The Comfortable Driving Behavior and New Model. In normal circumstance, drivers prefer to drive cars keeping a comfortable space headway and velocity; the cars will not accelerate with a much high acceleration. That is to say, if the space headways and velocities deviate from the comfortable driving conditions, the vehicles will accelerate or decelerate to make the cars keep the comfortable space headways and velocities.

According to the above analyses, we know the unrealistically high accelerations exist in the previous models, and the maximum acceleration is only related to value of the sensitivity coefficient; that is, the higher the value of $\kappa$ is, the higher the acceleration of the leading car is and vice versa. Therefore, we should decrease the value of the coefficient $\kappa$ for decreasing the acceleration; but, at the same time, the lower the value of $\kappa$ is, the lower the kinematic wave speed is, which are contradictory. Since the GFM has the problem of lower kinematic wave speed (cf. Figure 4(a) and Table 1), we add a new acceleration term into GFM for increasing the kinematic wave speed, and it should be effective when the velocity of the following vehicle is smaller than that of the leading vehicle, which is

$$
\frac{\Delta v_{n} \Theta\left(\Delta v_{n}\right)}{\tau_{n}^{\prime \prime}} e^{-\left[s\left(v_{n}\right)-s_{n}\right] / R_{n}^{\prime}}
$$

where $\tau_{n}^{\prime \prime}$ represents the acceleration time and other parameters are the same as GFM. So, we develop the improved generalized force model (IGFM):

$$
\begin{aligned}
\frac{\mathrm{d} v_{n}}{\mathrm{~d} t}= & \kappa\left[V\left(s_{n}, v_{n}\right)-v_{n}\right]+\lambda_{1} \Delta v_{n} \Theta\left(-\Delta v_{n}\right) \\
& +\lambda_{2} \Delta v_{n} \Theta\left(\Delta v_{n}\right),
\end{aligned}
$$

where $\lambda_{1}$ and $\lambda_{2}$ are deceleration and acceleration term coefficients with the following form:

$$
\begin{aligned}
& \lambda_{1}=\frac{1}{\tau_{n}^{\prime}} e^{-\left[s_{n}-s\left(v_{n}\right)\right] / R_{n}^{\prime}}, \\
& \lambda_{2}=\frac{1}{\tau_{n}^{\prime \prime}} e^{-\left[s\left(v_{n}\right)-s_{n}\right] / R_{n}^{\prime}} .
\end{aligned}
$$

In the IGFM, the comfortable driving behavior can be reflected by the two coefficients $\lambda_{1}$ and $\lambda_{2}$ depicted in Figure 3, where $s_{c}$ and $v_{c}$ are defined as the comfortable driving headway distance and the comfortable driving velocity, respectively.

As shown in Figure 3, the coefficients have the following properties.

(1) The deceleration term coefficient $\lambda_{1}$ increases gradually with the growing of space headway, and the acceleration term coefficient $\lambda_{2}$ is just the opposite. In Figure 3(a), when $d_{n}<s_{n}<s_{c}, \lambda_{1}>\lambda_{2}$, which means if the space headway is less than $s_{c}$, the reaction intensity of deceleration $\lambda_{1}$ is stronger than that of acceleration $\lambda_{2}$; that is, the driver prefers to decelerate for increasing the space headway; when $s_{c}<s_{n}<$ $R_{n}^{\prime}, \lambda_{1}<\lambda_{2}$, which means if the space headway is larger than $s_{c}$, the reaction intensity of deceleration is weaker than that of acceleration, and the driver prefers to accelerate for decreasing the space headway. These can make the cars always tend to run with the comfortable distance.

(2) The deceleration term coefficient $\lambda_{1}$ increases gradually with growing velocity, and the acceleration term coefficient $\lambda_{2}$ decreases, which is because the car's power is fixed and its acceleration decreases with the velocity growing. As shown in Figure 3(b), when $0<$ $v_{n}<v_{c}, \lambda_{1}<\lambda_{2}$, which means the vehicle prefers to accelerate when its velocity is lower than $v_{c}$; when $v_{c}<v_{n}<v_{m}, \lambda_{1}>\lambda_{2}$, which indicates that the vehicle prefers to decelerate when its speed is higher than $v_{c}$. These can make the cars always inclined to run at the comfortable velocity. 
2.2. The Calculation Methods of the Comfortable Distance and Velocity. From Figure 3(a), one can find when the headway distance $s_{n}$ is equal to the comfortable distance $s_{c}$ and the coefficient $\lambda_{1}=\lambda_{2}$; that is,

$$
\frac{e^{-\left[s_{c}-s\left(v_{n}\right)\right] / R_{n}^{\prime}}}{\tau_{n}^{\prime}}=\frac{e^{-\left[s\left(v_{n}\right)-s_{c}\right] / R_{n}^{\prime}}}{\tau_{n}^{\prime \prime}} .
$$

Take logarithm on both sides of equation; one can get

$$
\frac{\left[s_{c}-s\left(v_{n}\right)\right]}{R_{n}^{\prime}}=\ln \frac{\tau_{n}^{\prime \prime}}{\tau_{n}^{\prime}}+\frac{\left[s\left(v_{n}\right)-s_{c}\right]}{R_{n}^{\prime}} .
$$

If we simplify the above equation, we can obtain the comfortable driving distance

$$
s_{c}=s\left(v_{n}\right)+\frac{R_{n}^{\prime}}{2} \ln \frac{\tau_{n}^{\prime \prime}}{\tau_{n}^{\prime}} .
$$

The comfortable driving velocity is determined by the optimal velocity according to the comfortable distance; that is,

$$
v_{c}=V\left(s_{c}, v_{c}\right) \text {. }
$$

From (12), the comfortable driving distance includes two parts: one is the velocity-dependent safe distance $s\left(v_{n}\right)$; the other is the adjustable distance depending on the accelerating and braking time. If $\tau_{n}^{\prime}=\tau_{n}^{\prime \prime}, \ln \left(\tau_{n}^{\prime \prime} / \tau_{n}^{\prime}\right)=0$; that is, the adjustable distance is zero and the comfortable driving distance $s_{c}=s\left(v_{n}\right)$. In general, the vehicle's capacity in deceleration is higher than that in acceleration, so the accelerating time $\tau_{n}^{\prime \prime}$ is greater than the braking time $\tau_{n}^{\prime}$ and $\ln \left(\tau_{n}^{\prime \prime} / \tau_{n}^{\prime}\right)>0$. Therefore, the comfortable driving distance is greater than the safe distance generally, which is consistent with the reality.

2.3. Linear Stability Analysis. Linear stability analysis can be conducted for IGFM. The steady state is that vehicles move with the uniform space headway $s_{e}$, and the corresponding velocity is $V\left(s_{e}, v_{e}\right)$. According to chapter 15 of [11], the stability criterion is given by

$$
V_{e}^{\prime}\left(s_{e}, v_{e}\right) \leq-\frac{a_{v}}{2}-a_{\Delta v}, \quad s_{e}=\frac{L}{N}
$$

This formula applies to the models whose acceleration function is of the form $\mathrm{d} v / \mathrm{d} t=a_{\text {mic }}\left(s_{\alpha}, v_{\alpha}, \Delta v_{\alpha}\right)$, where $V_{e}\left(s_{e}, v_{e}\right)$ is the equilibrium gap-velocity relation, and $a_{v}, a_{\Delta v}$ are the partial derivatives of the acceleration of the model; that is, $a_{v}=\partial a_{\text {mic }} / \partial v, a_{\Delta v}=\partial a_{\text {mic }} / \partial \Delta v, L$ is the length of road, and $N$ is the number of cars. For IGFM, the acceleration is described by (8), so we arrive at the partial derivatives

$$
\begin{aligned}
a_{v}= & \frac{\partial a_{\mathrm{mic}}}{\partial v} \\
= & k\left\{-\frac{V_{m}}{T_{n}} e^{-\left[s_{e}-s\left(v_{e}\right)\right] / R_{n}}-1\right\} \\
& +\lambda_{1}\left(s_{e}, v_{e}\right) \Theta(-\Delta v) \frac{T_{n}}{R_{n}^{\prime}} \\
& -\lambda_{2}\left(s_{e}, v_{e}\right) \Theta(\Delta v) \frac{T_{n}}{R_{n}^{\prime}}, \\
a_{\Delta v}= & \frac{\partial a_{\mathrm{mic}}}{\partial v} \\
= & \lambda_{1}\left(s_{e}, v_{e}\right) \Theta(-\Delta v)+\lambda_{2}\left(s_{e}, v_{e}\right) \Theta(\Delta v) .
\end{aligned}
$$

Inserting (15) into (14), we obtain the string stability criterion

$$
\begin{aligned}
& V_{e}^{\prime}\left(s_{e}, v_{e}\right) \\
& \leq- \frac{1}{2}\left\{k\left\{-\frac{v_{m}}{T_{n}} e^{-\left[s_{e}-s\left(v_{e}\right)\right] / R_{n}}-1\right\}\right. \\
&\left.+\lambda_{1}\left(s_{e}, v_{e}\right) \Theta(-\Delta v) \frac{T_{n}}{R_{n}^{\prime}}-\lambda_{2}\left(s_{e}, v_{e}\right) \Theta(\Delta v) \frac{T_{n}}{R_{n}^{\prime}}\right\} \\
&-\left[\lambda_{1}\left(s_{e}, v_{e}\right) \Theta(-\Delta v)+\lambda_{2}\left(s_{e}, v_{e}\right) \Theta(\Delta v)\right] \\
& \leq \frac{k}{2}\left\{\frac{v_{m}}{T_{n}} e^{-\left[s_{e}-s\left(v_{e}\right)\right] / R_{n}}+1\right\} \\
&-\lambda_{1}\left(s_{e}, v_{e}\right) \Theta(-\Delta v)\left(1+\frac{T_{n}}{2 R_{n}^{\prime}}\right) \\
&-\lambda_{2}\left(s_{e}, v_{e}\right) \Theta(\Delta v)\left(1-\frac{T_{n}}{2 R_{n}^{\prime}}\right),
\end{aligned}
$$

where $\Theta(x)=1$ if $x>0$, and $\Theta(x)=0$ if $x \leq 0$. The stable condition of IGFM, especially, is

$$
V_{e}^{\prime}\left(s_{e}, v_{e}\right) \leq\left\{\begin{aligned}
\frac{k}{2}\left\{\frac{v_{m}}{T_{n}} e^{-\left[s_{e}-s\left(v_{e}\right)\right] / R_{n}}+1\right\} \\
-\lambda_{1}\left(s_{e}, v_{e}\right)\left(1+\frac{T_{n}}{2 R_{n}^{\prime}}\right) \quad \text { if } \Delta v<0 \\
\frac{k}{2}\left\{\frac{v_{m}}{T_{n}} e^{-\left[s_{e}-s\left(v_{e}\right)\right] / R_{n}}+1\right\} \\
\quad-\lambda_{2}\left(s_{e}, v_{e}\right)\left(1-\frac{T_{n}}{2 R_{n}^{\prime}}\right) \quad \text { if } \Delta v>0 .
\end{aligned}\right.
$$

As for FVDM, $\lambda$ is a constant and $V(s)$ do not depend on the velocity $v$, so its stable condition is

$$
V^{\prime}<\frac{\kappa}{2}+\lambda
$$




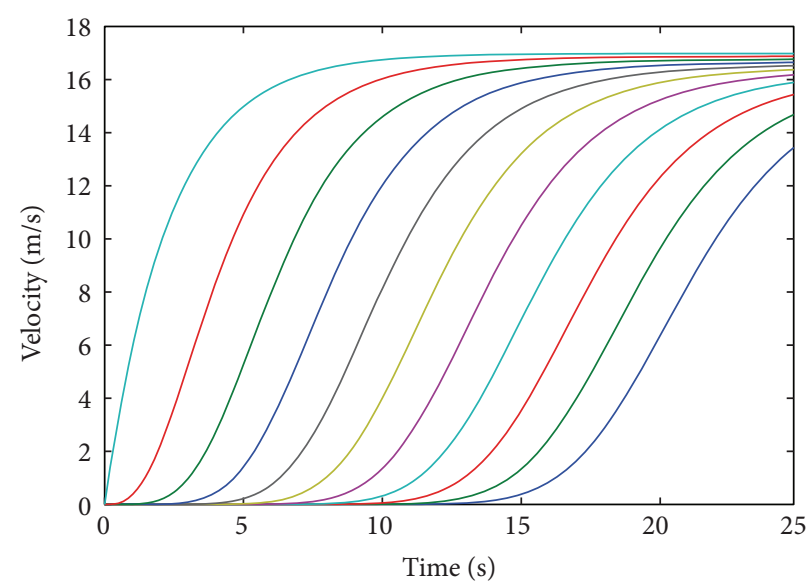

(a)

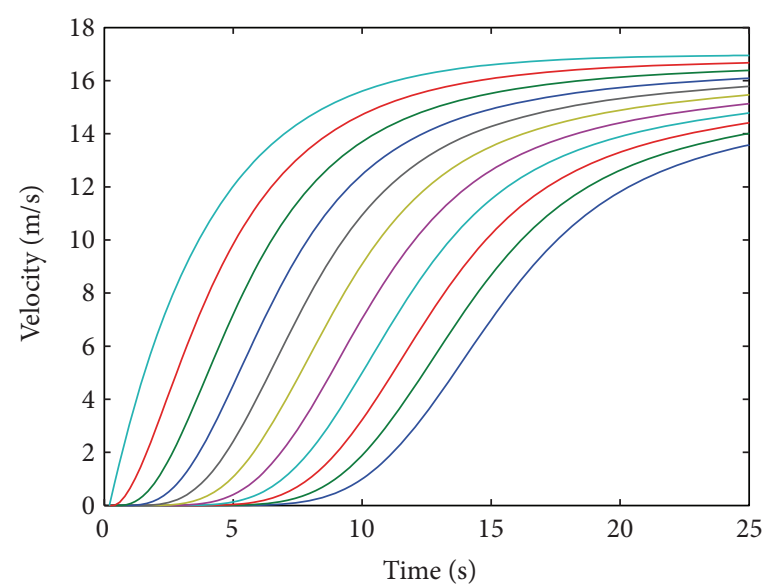

(b)

FIGURE 4: Variation of velocities of all vehicles starting from a traffic signal. (a) GFM. (b) IGFM.

As for OVM, $\lambda=0$, so its stable condition is

$$
V^{\prime}<\frac{\kappa}{2}
$$

Comparing with the results of IGFM, FVDM, and OVM, one can find that they are essentially the same. If $\lambda_{1}=\lambda_{2}=\lambda$, we can get the FVDM; if $\lambda_{1}=\lambda_{2}=0$, IGFM is reduced to OVM.

\section{Simulation}

In this section, we carry out the simulations to investigate whether IGFM can overcome the shortcomings of previous models and analyze the improvements of IGFM compared with GFM and OVM. In simulations, we set $\tau_{n}^{\prime \prime}=1.5 \mathrm{~s}$, and the values of other parameters are the same as those in [16]; that is, $v_{n}^{0}=16.98 \mathrm{~m} / \mathrm{s}, \kappa=0.25, d_{n}=1.38 \mathrm{~m}, T_{n}=0.74 \mathrm{~s}$, $\tau_{n}^{\prime}=1.5 \mathrm{~s}, \tau_{n}^{\prime \prime}=0.77 \mathrm{~s}, R_{n}=5.59 \mathrm{~m}, R_{n}^{\prime}=98.78 \mathrm{~m}$, and $l_{c}=5 \mathrm{~m}$. We use discrete velocity updating equation which can be deduced by the Euler scheme; that is,

$$
\begin{aligned}
v_{n}(t+\Delta t) \\
=v_{n}(t) \\
\quad+\Delta t\left\{\kappa\left[V\left(s_{n}, v_{n}\right)-v_{n}\right]+\lambda\left[v_{n+1}(t)-v_{n}(t)\right]\right\},
\end{aligned}
$$

where $\lambda=\lambda_{1}$ when $\Delta v_{n} \geq 0 ; \lambda=\lambda_{2}$ when $\Delta v_{n}<0$, and the calculation time interval $\Delta t=0.2 \mathrm{~s}$.

Firstly, the delay time $\delta t$ of car motion and the kinematic wave speed $C_{j}$ at jam density are examined to verify whether the unrealistic high acceleration exists in the IGFM. We carry out the simulation as in [17]. First a traffic signal is yellow and all cars (11 cars) are waiting with the uniform headway $6.38 \mathrm{~m}$, at which the optimal velocity is zero. Then, at time $t=0$, the signal changes to green and cars start.

The simulation results are shown in Figure 4 and Table 1. From Figure 4, one can see that the cars in IGFM accelerate more quickly than those in GFM, which means the delay time of IGFM is shorter than that of GFM. As shown in Table 1, the delay time $\delta t=1.22 \mathrm{~s}$ and $C_{j}=18.84 \mathrm{~km} / \mathrm{h}$ in IGFM, while $\delta t=1.76 \mathrm{~s}$ and $C_{j}=12.96 \mathrm{~km} / \mathrm{h}$ in GFM. Bando et al. [21] claimed that the observed $\delta t$ is of the order of $1 \mathrm{~s}$, and del Castillo and Benítez [22] indicated that $C_{j}$ ranges between 17 and $23 \mathrm{~km} / \mathrm{h}$. Therefore, we can see that the GFM is poor in anticipating the two parameters, but the IGFM overcomes the disadvantages and it can describe the delay time and kinematic speed more reasonably. In addition, the acceleration is a decreasing function of the speed and the vehicle can accelerate towards a desired speed. Therefore, the new model satisfies one of the model conditions which is described in [11].

Figure 5(a) shows the variation of acceleration under the case that two successive cars are initially at rest, and the leading car is unobstructed. At $t=0$, all cars start up according to the OVM, GFM, and IGFM, respectively. From Figure 4(a), it can be seen that the maximal accelerations are $4.2 \mathrm{~m} / \mathrm{s}^{2}, 12.46 \mathrm{~m} / \mathrm{s}^{2}$, and $6.96 \mathrm{~m} / \mathrm{s}^{2}$ in IGFM, OVM, and GFM. The empirical acceleration should be limited to $4 \mathrm{~m} / \mathrm{s}^{2}$, so IGFM is more consistent with reality than the existing models. In Figure 5(b), the solid line denotes the accelerations of all vehicles in IGFM, and the dashed lines represent those of the first and the last follower in OVM and GFM. We can see that the vehicles in IGFM accelerate more quickly than those in OVM and GFM, which is the reason that kinematic speed is larger and the delay time is shorter in IGFM than those in other two models.

Secondly, we simulate the vehicles' behaviors under two decelerating cases to demonstrate whether there is an accident that happened in IGFM. One is the free decelerating case; the other is the urgent braking case. In this paper, the free decelerating case can be defined as follows: the leading car stops all the time, and the follower moves freely with the initial speed $16.98 \mathrm{~m} / \mathrm{s}$; the headway between them is $120 \mathrm{~m}$. Simulation results are shown in Figure 6. One can see that the maximal deceleration rates are $-6.51 \mathrm{~m} / \mathrm{s}^{2},-10.1 \mathrm{~m} / \mathrm{s}^{2}$, and $-9.4 \mathrm{~m} / \mathrm{s}^{2}$ in OVM, GFM, and IGFM, respectively, and 


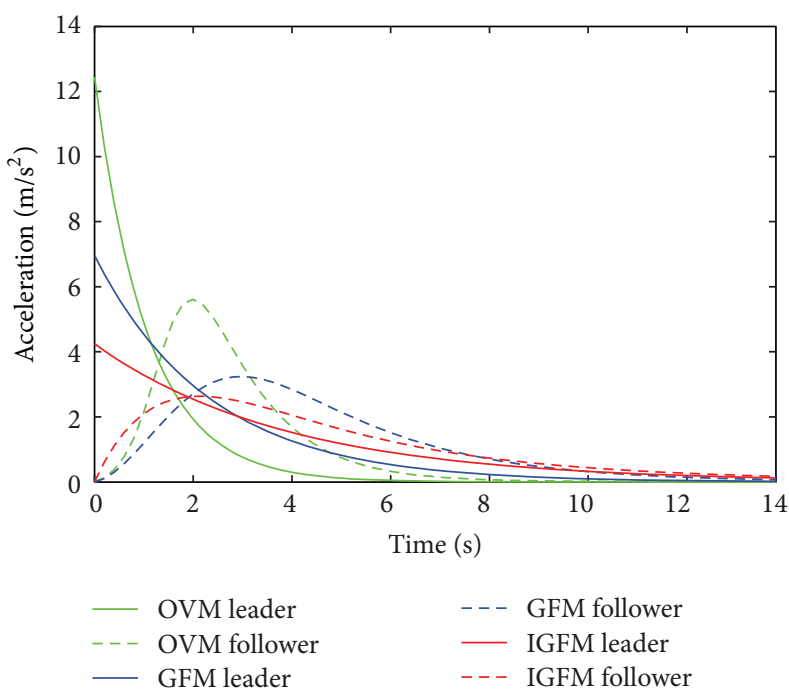

(a)

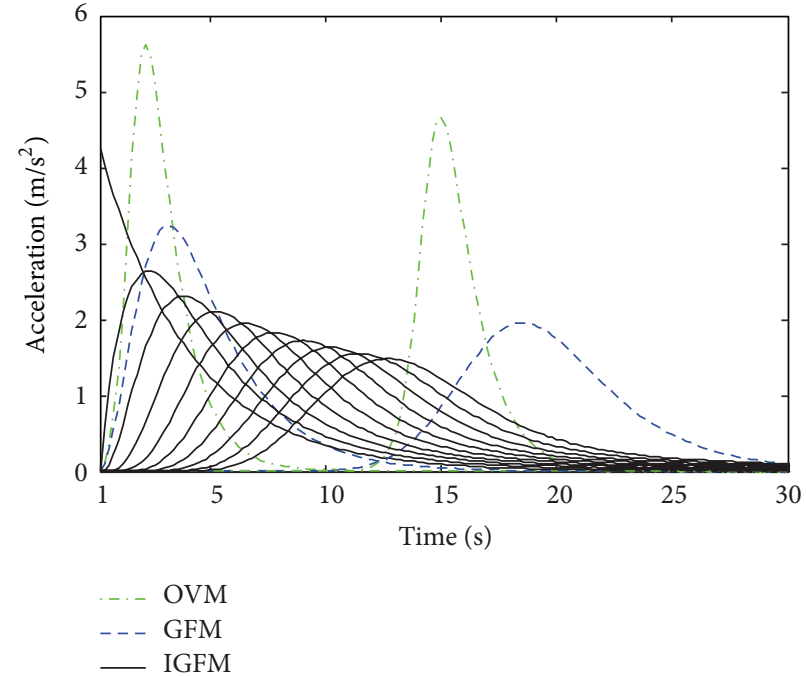

(b)

FIGURE 5: (a) Acceleration of an unobstructed car and of a following car according to OVM, GFM, and IGFM; (b) accelerations of all cars in IGFM.

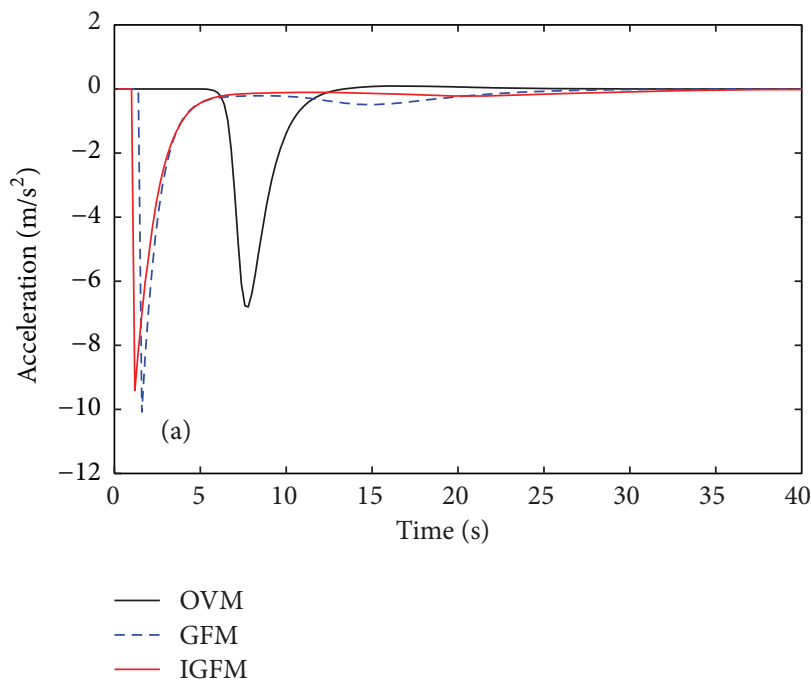

(a)

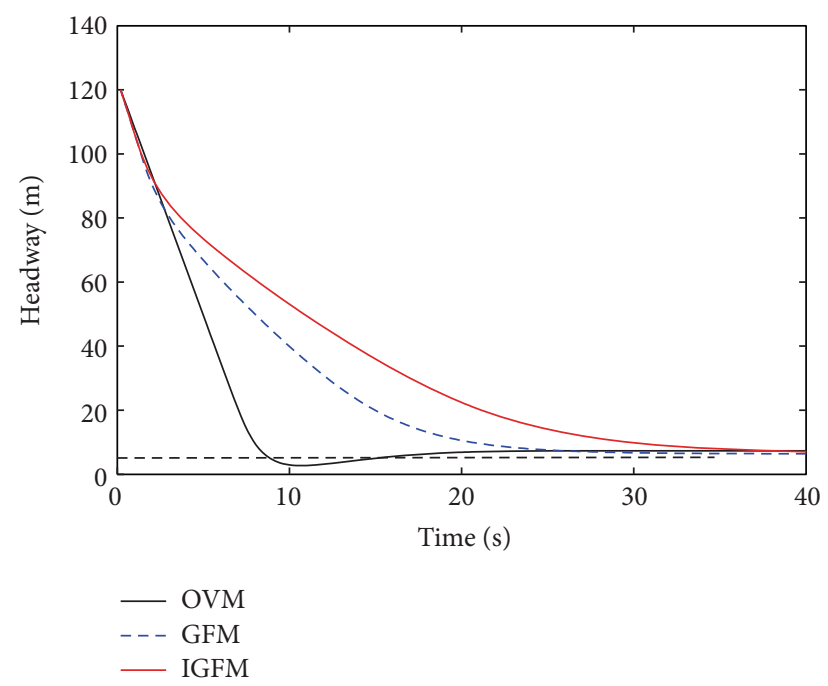

(b)

FIGURE 6: Simulations in the OVM, GFM, and IGFM under the free decelerating case. (a) Deceleration of the follower; (b) headway distance of the follower.

the deceleration rate in IGFM is less than that in GFM. One can also note that deceleration rate in OVM is the least; however, the space headway is lower than $5 \mathrm{~m}$ as shown in Figure 6(b), which means crash occurs. As for the IGFM, no crash occurs because the headway is higher than $5 \mathrm{~m}$, and no backward movement occurs because the acceleration of IGFM approaches zero when the gap comes to the minimum gap, so the IGFM can describe the free decelerating case successfully.

We simulate the vehicles' behaviors under the urgent braking case referred to [23]. The urgent braking case can be defined as: a situation that the preceding car decelerates strongly, if two successive cars move forward with much small headway-distance, for example, a freely moving car decelerates drastically for an accident in front or the red traffic light at an intersection. According to (4), the safe headway distance between two successive cars is $14 \mathrm{~m}$ when they run at maximal speed $v_{n}^{0}=16.98 \mathrm{~m} / \mathrm{s}$. So the simulation under such situation is carried out in the following: two successive cars move with the same speed $16.98 \mathrm{~m} / \mathrm{s}$ at the initial time $t=0$ and the space headway is $14 \mathrm{~m}$; then leading car decelerates suddenly with the deceleration $-6 \mathrm{~m} / \mathrm{s}^{2}$ until it stops completely. The leading car remains standing for $7 \mathrm{~s}$ before accelerating back to its original speed. The simulation 


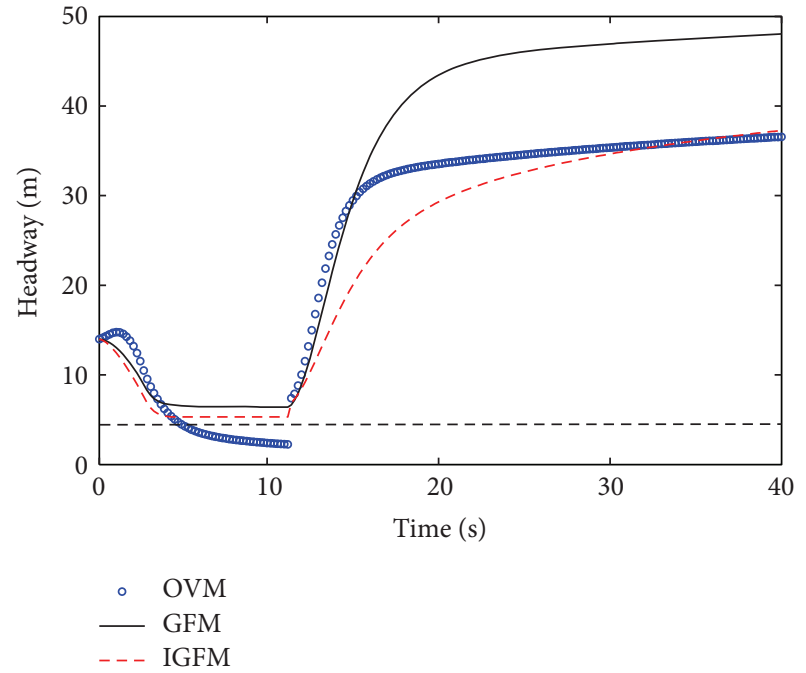

(a)

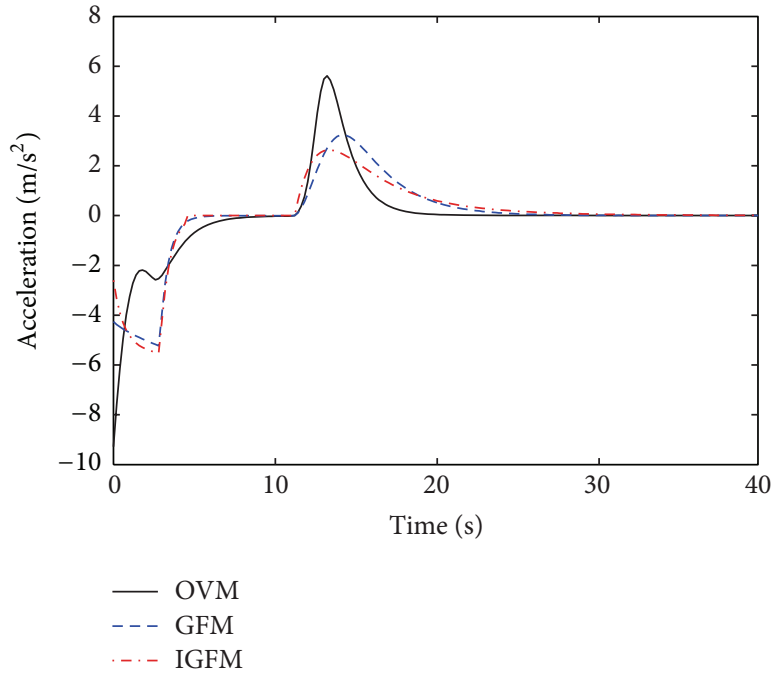

(b)

FIGURE 7: Simulations in the OVM, GFM, and IGFM under an urgent braking case. (a) Space headway of the follower; (b) acceleration of the follower.

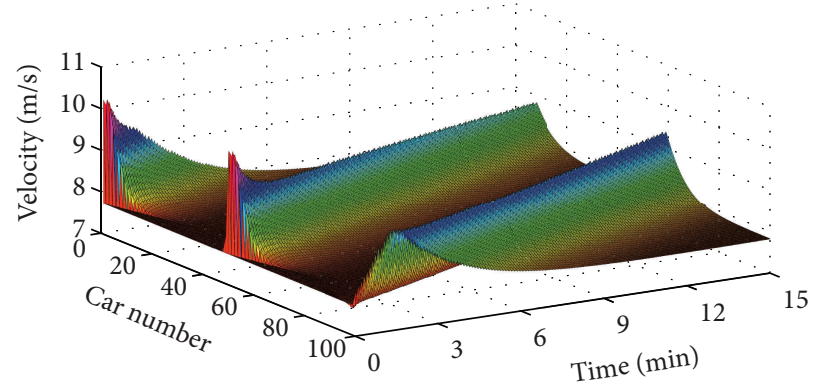

(a)

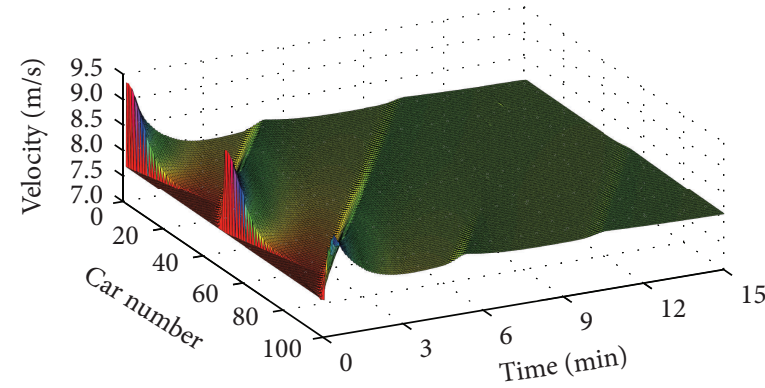

(b)

FIGURE 8: Spatiotemporal velocity plots of traffic states. (a) GFM. (b) IGFM.

results are shown in Figure 7(a), which denotes the variation of space headway of the following car. One can see that the space headway is lower than $5 \mathrm{~m}$ in OVM; this indicates the leading car collides with the following one. Instead, the space headways are always larger than $5 \mathrm{~m}$ in GFM and IGFM due to the fact that the followers can adjust their speeds timely; that is, the IGFM can avoid the accident.

Thirdly, a simulation is carried out to observe the traffic dynamics in IGFM. There are $N=100$ cars running on a road with the length $L=1600 \mathrm{~m}$, under a periodic boundary condition. We set the initial disturbance as

$$
\begin{aligned}
& x_{1}(0)=10 \mathrm{~m}, \\
& s_{1}(0)=s_{51}(0)=40.5 \mathrm{~m}, \\
& s_{i}(0)=10.5 \mathrm{~m}, \quad \text { for } i \neq 1,51, \\
& v_{i}(0)=V\left(s_{i}\right),
\end{aligned}
$$

where $s_{i}$ denotes the space headway, and other parameters are the same as the previous simulation. Here, the simulation is carried out $900 \mathrm{~s}$. According to (17), the initial disturbance is unstable for GFM, but stable for IGFM.

Figure 8 shows the spatiotemporal velocity of traffic states, according to GFM and IGFM respectively. As shown in Figure $8(\mathrm{a})$, one can learn that the amplitude of velocity remains unchanged with the evolution of time in GFM, which indicates the traffic state is always unstable; but, in Figure 8 (b), the amplitude of velocity decreases gradually in IGFM, which means the traffic gradually reaches to a stable state as time going on. At $t=900 \mathrm{~s}$, the velocities of cars in IGFM are about $8.1 \mathrm{~m} / \mathrm{s}$. In addition, the propagation velocity of disturbance in IGFM is faster than that in GFM; this effect is attributed to the fact that the drivers' reaction in IGFM is rapider, and it can weaken the velocity fluctuation caused by disturbance. These suggest that IGFM has a stronger capability of resisting disturbance and better stability than GFM. The results are consistent with the stability analysis.

In order to get further dynamic properties of vehicles, the headway-velocity plots for GFM and IGFM are depicted in Figure 9. As shown in Figure 9, the headway ranges from $15.5 \mathrm{~m}$ to $18.3 \mathrm{~m}$ in GFM and from $15.5 \mathrm{~m}$ to $16.7 \mathrm{~m}$ in IGFM; 


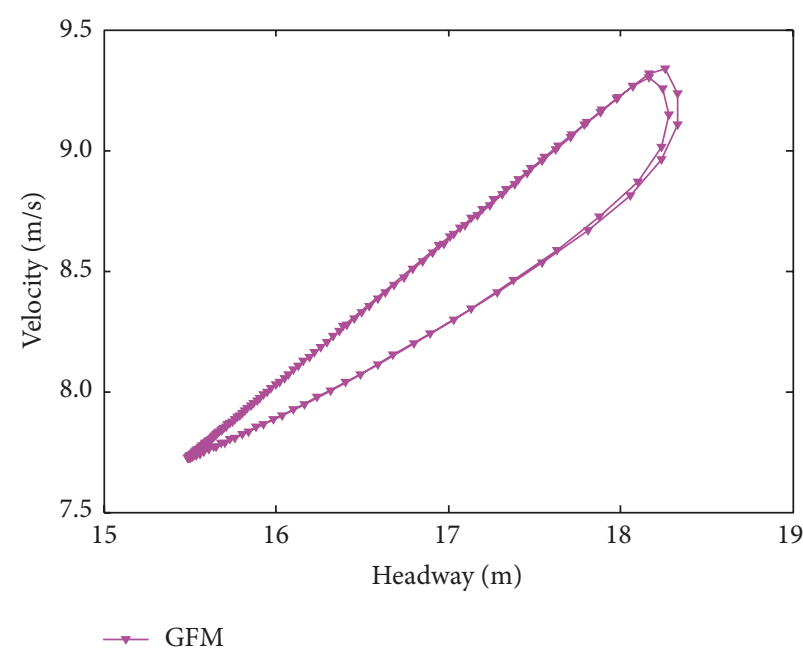

(a)

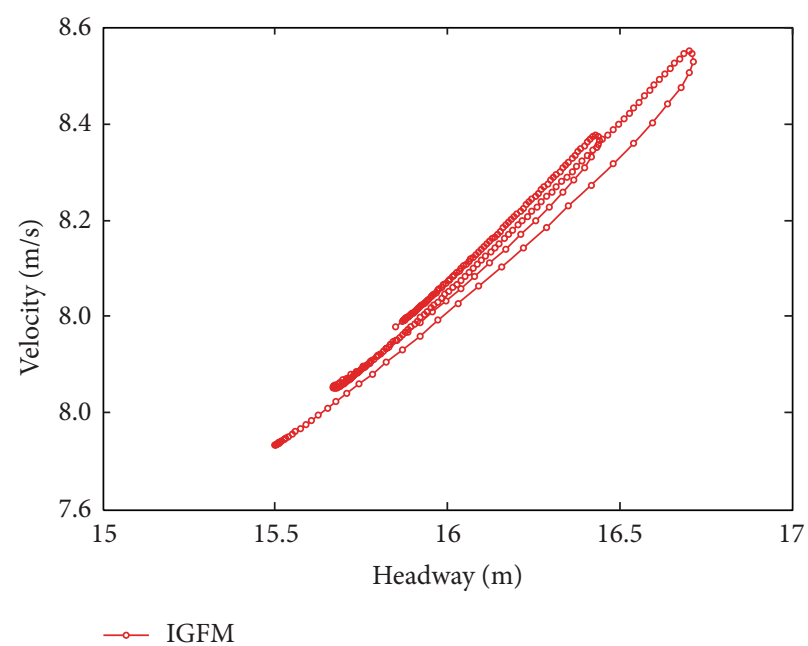

(b)

FIgURE 9: Headway-velocity plots. (a) GFM; (b) IGFM.

the velocity ranges from $7.7 \mathrm{~m} / \mathrm{s}$ to $9.4 \mathrm{~m} / \mathrm{s}$ in GFM and from $7.82 \mathrm{~m} / \mathrm{s}$ to $8.45 \mathrm{~m} / \mathrm{s}$ in IGFM. It is obvious that the headway and velocity fluctuation in GFM are not weakened, but they decrease in IGFM, which suggests that the disturbance is disappearing and the traffic state is changing stably; they always remain unchanged in GFM. Furthermore, a "hysteresis loop" is clearly observed in GFM but not in IGFM, which is an effect we ascribe to the acceleration term coefficient in IGFM and it reduces the symmetry of acceleration and deceleration capacities. This is exactly the reason that drivers in IGFM are more sensitive than those in GFM and the IGFM has a better stability.

\section{Conclusions}

Through introducing the acceleration term into the GFM, the improved generalized force model (IGFM) is presented considering the comfortable driving behavior. In addition, the calculation methods of the comfortable distance and velocity are obtained. The new model can predict the kinematic speed at jam density and the delay time of car motion more correctly, and it can exactly describe the drivers' behavior under the free decelerating and urgent cases, where no collision occurs. The more important improvement is that the IGFM reduces the maximal acceleration of leading car to $4.2 \mathrm{~m} / \mathrm{s}$ when it starts up with no obstructions, which is much close to the reality. However, the unrealistic high acceleration occurs in the previous models. Linear analysis is used to investigate the properties of the new model and the stability condition is obtained. Moreover, the simulation results reveal that IGFM's stability has improved.

\section{Conflict of Interests}

The authors declare that there is no conflict of interests regarding the publication of this paper.

\section{Acknowledgments}

This research was funded by the National Basic Research Program of China (2012CB725406), the National Natural Science Foundation of China (71131001), and the National Natural Science Foundation of China (71390332).

\section{References}

[1] D. Helbing, "Traffic and related self-driven many-particle systems," Reviews of Modern Physics, vol. 73, no. 4, pp. 1067-1141, 2001.

[2] B. S. Kerner, S. L. Klenov, and D. E. Wolf, "Cellular automata approach to three-phase traffic theory," Journal of Physics A: Mathematical and General, vol. 35, no. 47, pp. 9971-10013, 2002.

[3] Y. S. Qian, X. M. Shao, J. W. Zeng, and M. Wang, "An improved cellular automaton model with the consideration of a multi-point tollbooth," Physica A: Statistical Mechanics and Its Applications, vol. 392, no. 23, pp. 5874-5878, 2013.

[4] J. W. Zeng, Y. S. Qian, D. J. Xu, Z. L. Jia, and Z. D. Huang, "Impact of road bends on traffic flow in a single-lane traffic system," Mathematical Problems in Engineering, vol. 2014, Article ID 218465, 6 pages, 2014.

[5] K. Nagel and M. Schreckenberg, "A cellular automaton model for freeway traffic," Journal de Physique I, vol. 2, no. 12, pp. 2221$2229,1992$.

[6] H. X. Ge, S. Q. Dai, L. Y. Dong, and Y. Xue, "Stabilization effect of traffic flow in an extended car-following model based on an intelligent transportation system application," Physical Review E: Statistical, Nonlinear, and Soft Matter Physics, vol. 70, no. 6, Article ID 066134, 2004.

[7] T. Q. Tang and H. J. Huang, "Continuum models for freeways with two lanes and numerical tests," Chinese Science Bulletin, vol. 49, no. 19, pp. 2097-2104, 2004.

[8] H.-X. Ge, X.-P. Meng, J. Ma, and S.-M. Lo, "An improved carfollowing model considering influence of other factors on traffic jam," Physics Letters A, vol. 377, no. 1-2, pp. 9-12, 2012. 
[9] W. Guan, S.-Y. He, and J.-H. Ma, "Review on traffic flow phenomena and theory," Journal of Transportation Systems Engineering and Information Technology, vol. 12, no. 3, pp. 90-97, 2012.

[10] T. Q. Tang, H. J. Huang, S. G. Zhao, and H. Y. Shang, "A new dynamic model for heterogeneous traffic flow," Physics Letters, Section A: General, Atomic and Solid State Physics, vol. 373, no. 29, pp. 2461-2466, 2009.

[11] M. Treiber and A. Kesting, Traffic Flow Dynamics: Data, Models and Simulation, Springer, Berlin, Germany, 2013.

[12] A. Reuschel, "Vehicle movements in a platoon," Österreichisches Ingenieur-Archiv, vol. 4, pp. 193-215, 1950.

[13] L. A. Pipes, "An operational analysis of traffic dynamics," Journal of Applied Physics, vol. 24, no. 3, pp. 274-281, 1953.

[14] G. F. Newell, "Nonlinear effects in the dynamics of car flowing," Operations Research, vol. 9, no. 2, pp. 209-229, 1961.

[15] M. Bando, K. Hasebe, K. Nakanishi, A. Nakayama, A. Shibata, and Y. Sugiyama, "Phenomenological study of dynamical model of traffic flow, Journal de Physique I, vol. 5, no. 11, pp. 1389-1399, 1995.

[16] D. Helbing and B. Tilch, "Generalized force model of traffic dynamics," Physical Review E-Statistical Physics, Plasmas, Fluids, and Related Interdisciplinary Topics, vol. 58, no. 1, pp. 133138, 1998.

[17] R. Jiang, Q. S. Wu, and Z. J. Zhu, "Full velocity difference model for a car-following theory," Physical Review E-Statistical, Nonlinear, and Soft Matter Physics, vol. 64, no. 1, Article ID 017101, 4 pages, 2001.

[18] H. X. Gong, H. C. Liu, and B.-H. Wang, "An asymmetric full velocity difference car-following model," Physica A, vol. 387, no. 11, pp. 2595-2602, 2008.

[19] H. Xu, H. C. Liu, and H. X. Gong, "Modeling the asymmetry in traffic flow (a): microscopic approach," Applied Mathematical Modelling, vol. 37, no. 22, pp. 9431-9440, 2013.

[20] S. Yu, Q. Liu, and X. Li, "Full velocity difference and acceleration model for a car-following theory," Communications in Nonlinear Science and Numerical Simulation, vol. 18, no. 5, pp. 1229-1234, 2013.

[21] M. Bando, K. Hasebe, K. Nakanishi, and A. Nakayama, "Analysis of optimal velocity model with explicit delay," Physical Review E, vol. 58, no. 5, pp. 5429-5435, 1998.

[22] J. M. del Castillo and F. G. Benítez, "On the functional form of the speed-density relationship-I: general theory," Transportation Research Part B, vol. 29, no. 5, pp. 373-389, 1995.

[23] X. Zhao and Z. Gao, "A new car-following model: full velocity and acceleration difference model," The European Physical Journal B, vol. 47, no. 1, pp. 145-150, 2005. 


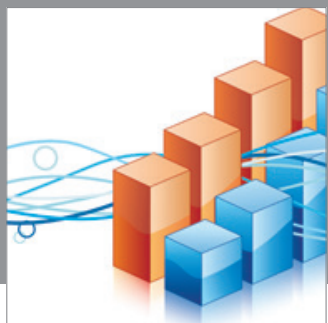

Advances in

Operations Research

mansans

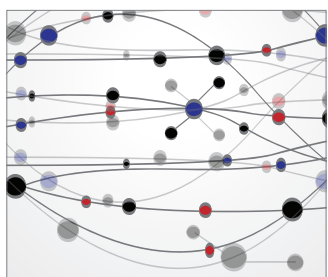

The Scientific World Journal
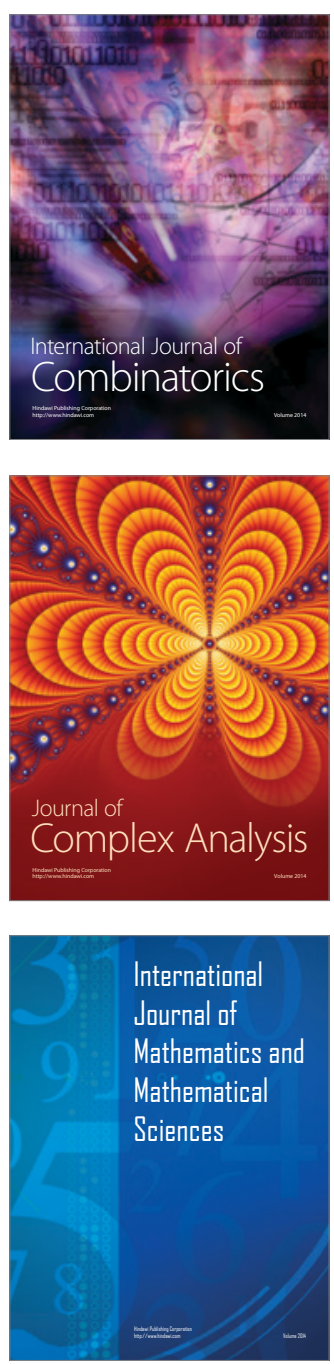
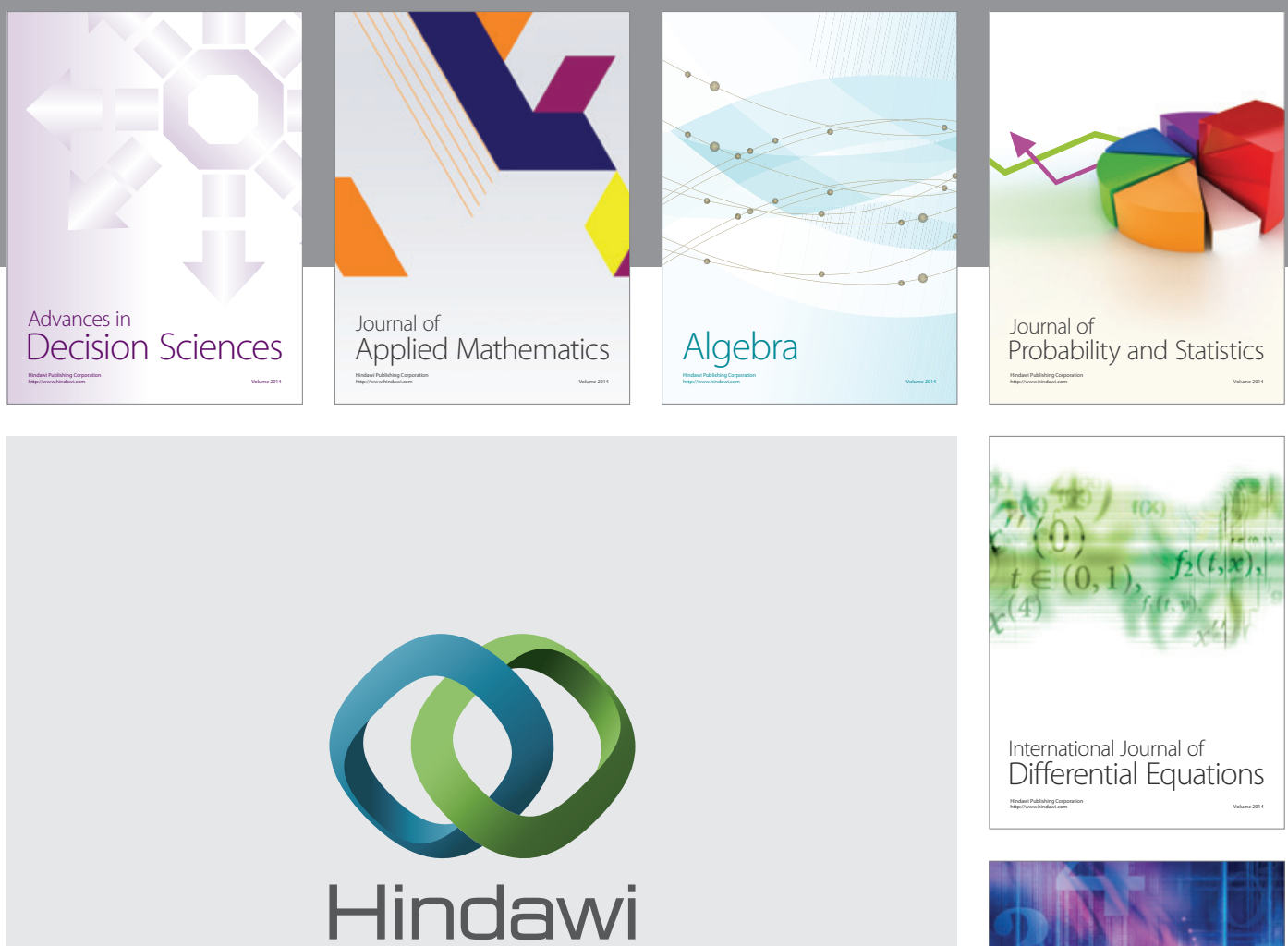

Submit your manuscripts at http://www.hindawi.com
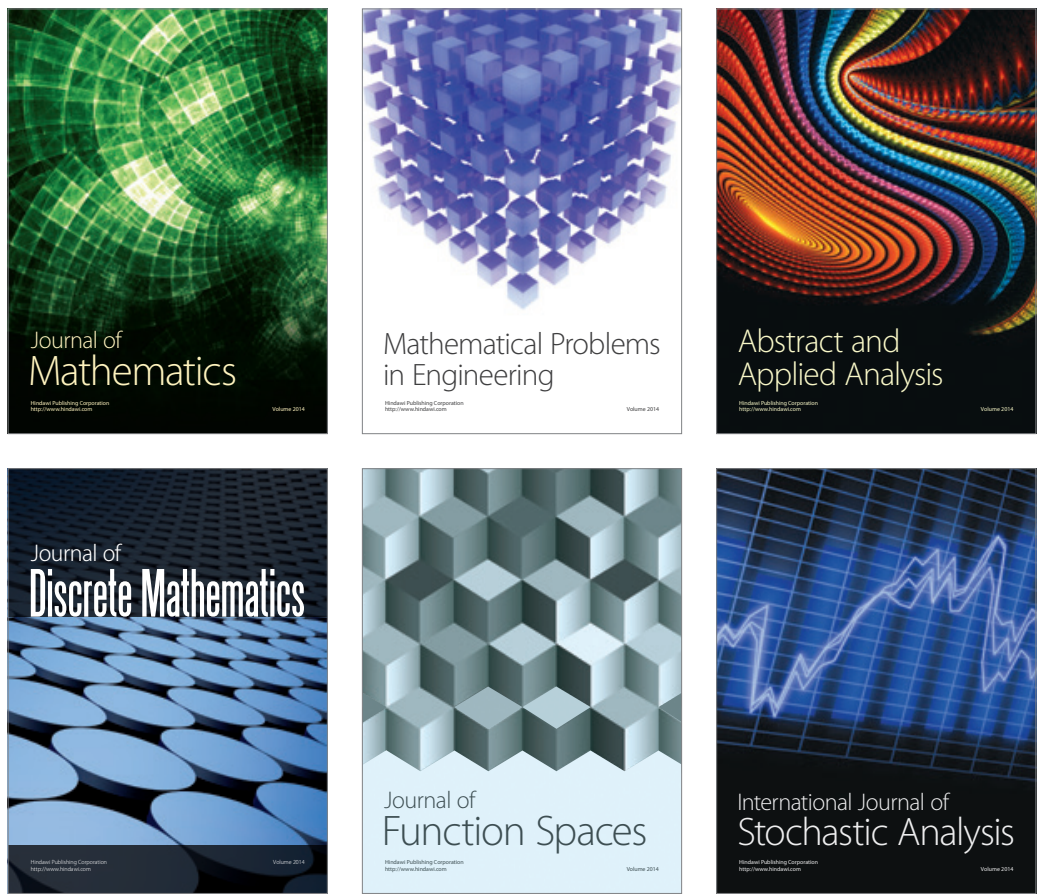

Journal of

Function Spaces

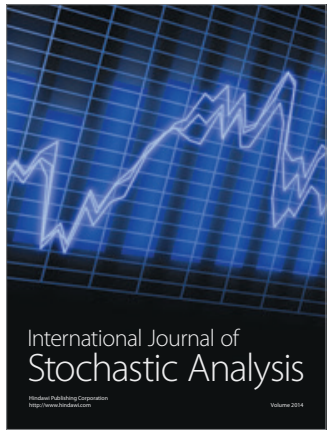

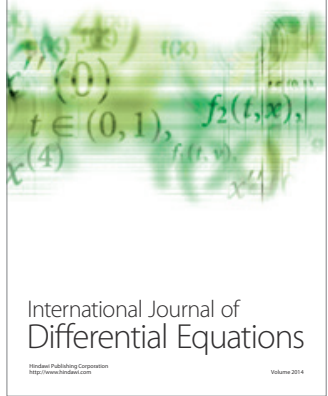
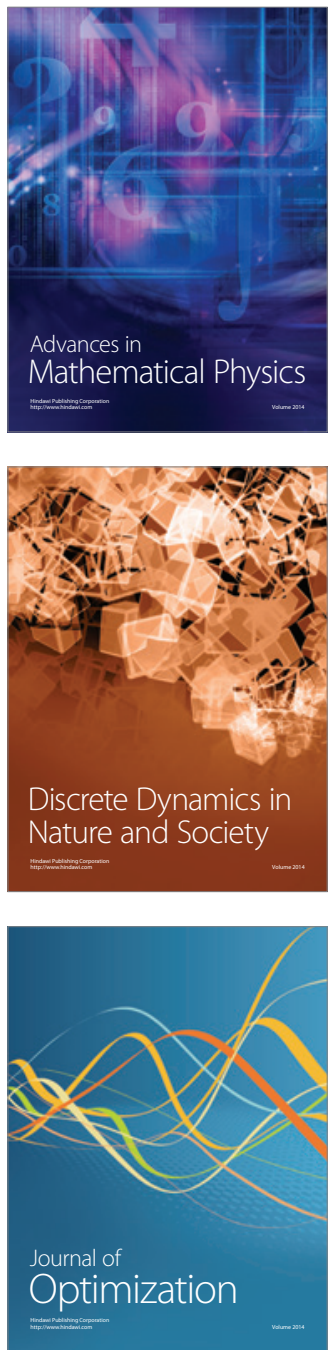